\title{
A Simple Return Generating Model in Discrete Time: Implications and Potential Applications for Market Efficiency Testing and Technical Analysis
}

\author{
Alexandros E. Milionis ${ }^{1,2}$ \\ ${ }^{1}$ Department of Mathematics, University of the Aegean, Karlovassi, Greece \\ ${ }^{2}$ Bank of Greece, Athens, Greece \\ Email: amilionis@aegean.gr
}

How to cite this paper: Milionis, A.E. (2019) A Simple Return Generating Model in Discrete Time: Implications and Potential Applications for Market Efficiency Testing and Technical Analysis. Theoretical Economics Letters, 9, 973-985.

https://doi.org/10.4236/tel.2019.94063

Received: January 9, 2019

Accepted: April 19, 2019

Published: April 22, 2019

Copyright $\odot 2019$ by author(s) and Scientific Research Publishing Inc. This work is licensed under the Creative Commons Attribution International License (CC BY 4.0).

http://creativecommons.org/licenses/by/4.0/

\begin{abstract}
A linear return generating model is introduced. This model is a generalization in discrete time of the differential equation describing dynamical systems in continuous time. The model is useful in its own right, as it provides a simplified, yet credible, quantitative description of the reality. Further, the model is used as a tool for a theoretical study of market efficiency testing. This is obtained by modelling certain market conditions under which new information is released and reflected in asset prices on the one hand, and, on the other hand, by recording what established econometric testing approaches conclude, about the hypothesis of market efficiency. Amongst others it is argued that, contrary to the general belief, theoretically a random walk in asset prices, under certain conditions, could be associated with profoundly inefficient markets. Furthermore, an enhancement of the battery of statistical tests for market efficiency is proposed by the potential application of specific forms of the suggested linear dynamic model and the possible advantages over the existing techniques are explained. Finally, market efficiency is discussed in its relation to Technical Analysis.
\end{abstract}

\section{Keywords}

Evolving Efficiency, Market Efficiency Testing, Random Walks, Return Generating Model, Return Predictability, Technical Analysis

\section{Introduction}

The concept of market efficiency is of paramount importance in modern finan- 
cial economics. According to the prevailing definition [1] a market is efficient if "prices fully reflect all available information". The classic categorization of the available information, first introduced by Roberts [2], discriminates efficiency as weak form (WFME), when the information set $\left(\Phi_{\mathrm{w}}\right)$ includes past prices, semi-strong form (SSFME), when the information set $\left(\Phi_{\mathrm{ss}}\right)$ includes all publicly available information, and strong form (SFME), when the information set $\left(\Phi_{s}\right)$ includes all, public or private, information (see also Elton et al., 2007). According to this classification, it is obvious that: $\Phi_{\mathrm{w}} \subseteq \Phi_{\mathrm{ss}} \subseteq \Phi_{\mathrm{s}}$, hence, SFME entails SSFME and SSFME entails WFME, but not vice versa. However, there exists a lot of debate about the definition of market efficiency itself, [3] [4] [5] [6] [7] as well as about its classification and testing. Although initially the framework used for the empirical testing of market efficiency was the one of random walk in conjunction with constant expected returns, it was recognized that due to the well known joint hypothesis problem (market efficiency-asset pricing model), univariate models less restrictive that the random walk, allowing for interdependence in past returns, do not necessarily entail inefficiency, if expected returns are not assumed constant [8]. This was possibly among the reasons for which Fama [9] suggested some changes in the terminology and coverage for the three classes of market efficiency: instead of weak-form tests he suggested the more general tests for "return predictability", which, in addition to past returns, host tests which may include variables related to firm characteristics, market characteristics and the time of the year. Cross sectional predictability of returns with the possible use of an asset pricing model could also be hosted. Further, he suggested that semi-strong form tests are restricted to studies of announcements, under the name "event studies". However, the fact by itself that, in most part of the literature, it is the original terminology that continued to be used after 1991 [10] [11] [12] [13] [14], and it is still being used [15] [16], is a clear testimony that the revised taxonomy was not fully adopted. Some of the reasons may be the following: 1) the borderline between the information sets of the first and the second category in the revised taxonomy was somewhat unclear; 2) as, unquestionably, tests in all categories refer to return predictability, it is apparently wrong to use this term for the first category only; 3) though SSFME clearly entails WFME, there is a less clear ordering between the categories "event studies" and "return predictability". For this reason, it is the classical market efficiency classification which will be taken into consideration in this work.

It is further noted that although market efficiency was originally perceived as an on-and-off characteristic, i.e. in a static sense, more recently a gradually expanding body of research work appeared challenging this static character of efficiency by providing empirical evidence which is conducive to an evolving rather than static efficiency (see Lim and Brooks [17] and many references there in).

To reconcile the entailed time-varying return predictability, Daniel and Titman [18] introduced the term "adaptive efficiency" and Lo [19] [20] proposed the so-called Adaptive Markets Hypothesis. In an adaptively efficient market profit opportunities may be created, but if investors "learn" from the past price 
history such opportunities gradually disappear.

In either its static or evolving version, market efficiency depends directly on the way that new information is propagated and reflected in asset prices. Several authors have proposed models which express quantitatively how fast market-wide information is capitalised into asset prices (Grossman and Stiglitz [21], see also Hou and Moskowitz [22] and references there in). There is little doubt, however, that as regards to the mechanisms which determine the way that the flows of new information shape asset price movements, we really stand in front of a reality which is awesomely complex. Each of the models mentioned previously, is based on specific assumptions and, at best, throws light only on a facet of this complexity.

Bearing in mind that the mechanisms under which the release and assimilation of new information is reflected in asset prices is the cornerstone for the study of market efficiency the aim of this work is to introduce a relatively simple model broadly describing these mechanisms in a purely theoretical level. Such a model will subsequently be used as a tool for the study of market efficiency. Furthermore, the potential applicability of special forms of this model for the empirical testing of market efficiency is discussed.

The rest of the paper is structured as follows: In Section 2, a return generating model is introduced and commented upon. In Section 3, market efficiency testing is examined in conjunction with this model. In Section 4, some additional comments are made relating to market efficiency testing and existing empirical findings. Section 5 summarises and concludes the paper.

\section{A Simplified Discrete Time Return Generating Model}

\subsection{Conceptual Framework}

It will be assumed that asset returns are determined by the assimilation of the dynamic impact of various pieces of new information released by various information sources at equispaced time intervals. Without loss of generality, as far as the final conclusions of this work are concerned, it will be assumed that such impacts are linear, though this may not be the case in real markets [23]. The information sources will be modelled as indication series which broadly may be divided into two types: (a) random indicator series, which release new information in the form of a stream of independently and identically distributed (i.i.d. henceforth) random shocks; and (b) non-random indicator series which release new information occasionally at scattered times and at the nominal measurement level (e.g. a stock split announcement, an acquisition announcement, etc). It is noted that the assumption that type (a) indicator series are i.i.d. entails a framework of constancy of expected returns.

\subsection{Model Description}

The determination of asset returns under the stated conceptual framework can be mathematically formulated with the following stochastic difference equation: 


$$
\begin{aligned}
R_{t} & =\sum_{i=1}^{K} U_{i}(B) I_{i t}+\sum_{j=1}^{M} U_{j}^{\prime}(B) \nabla\left(I_{j t}^{*}\right)+\frac{\Theta(B)}{\Phi(B)} \varepsilon_{t} \\
& =\sum_{i=1}^{K} \frac{\omega_{i}(B) B^{b_{i}}}{\delta_{i}(B)} I_{i t}+\sum_{j=1}^{M} \frac{\omega_{j}^{\prime}(B) B^{b_{j}^{\prime}}}{\delta_{j}^{\prime}(B)} \nabla\left(I_{j t}^{*}\right)+\frac{\Theta(B)}{\Phi(B)} \varepsilon_{t}
\end{aligned}
$$

where:

$R_{t}$ are the observed returns;

$I_{i t}$ is the $\mathrm{i}^{\text {th }}$ random indicator series;

$K$ is the total number of random indicator series explicitly included in Equation (1);

$I_{j t}^{*}$ is the $\mathrm{j}^{\text {th }}$ non-random indicator;

$M$ is the total number of non-random indicators explicitly included in Equation (1);

$B$ is the so-called backward shift operator such that: $B^{k} I_{t}=I_{t-k}$;

$\nabla=(1-B)$ is the difference operator;

$$
U_{i}(B)=u_{i 0}+u_{i 1} B+u_{i 2} B^{2}+\cdots
$$

is a polynomial of infinite order in $B$, called the transfer function relating $R_{t}$ with the $\mathrm{i}^{\text {th }}$ random input;

$u_{i 1}, u_{i 2}, \cdots$ are the so-called impulse response weights;

$$
U_{j}^{\prime}(B)=u_{j 0}^{\prime}+u_{j 1}^{\prime} B+u_{j 2}^{\prime} B^{2}+\cdots
$$

is a polynomial of infinite order in $B$, called the transfer function relating $R_{t}$ with the $\mathrm{j}^{\text {th }}$ non-random input;

$$
\omega_{i}(B)=\omega_{i 0}-\omega_{i 1} B-\omega_{i 2} B^{2}-\cdots-\omega_{i s_{i}} B^{s_{i}}
$$

is a polynomial of finite order $s_{i}$ in $B$;

$$
\omega_{j}^{\prime}(B)=\omega_{j 0}^{\prime}-\omega_{j 1}^{\prime} B-\omega_{j 2}^{\prime} B^{2}-\cdots-\omega_{j s_{j}^{\prime}}^{\prime} B^{s_{j}^{\prime}}
$$

is a polynomial of finite order $s_{j}^{\prime}$ in $B$;

$$
\delta_{i}(B)=1-\delta_{i 1} B-\delta_{i 2} B^{2}-\cdots-\delta_{i r_{i}} B^{r_{i}}
$$

is a polynomial of finite order $r_{i}$ in $B$;

$$
\delta_{j}^{\prime}(B)=1-\delta_{j 1}^{\prime} B-\delta_{j 2}^{\prime} B^{2}-\cdots-\delta_{j r_{j}^{\prime}}^{\prime} B^{r_{j}^{\prime}}
$$

is a polynomial of finite order $r_{j}^{\prime}$ in $B$;

$b_{i}, b_{j}^{\prime}$ are the so-called dead time parameters representing time delays between a stimulus in the $\mathrm{i}^{\text {th }}$ random input (information source) and $\mathrm{j}^{\text {th }}$ non-random input (information source) respectively and the reaction in the output (asset returns);

$$
\Phi(B)=1-\varphi_{1} B-\cdots-\varphi_{p} B^{p}
$$

is the autoregressive polynomial of order $p$;

$$
\Theta(B)=1-\theta_{1} B-\cdots-\theta_{q} B^{q}
$$

is the moving average polynomial of order $q$; and $\varepsilon_{t}$ is a pure white noise process. 


\subsection{Clarifications and Comments}

Some explanation regarding the model expressed by Equation (1) may be necessary. At first it is noted that Equation (1) is a generalization in discrete time of the following differential equation:

$$
\left(1+\pi_{1} D+\cdots+\pi_{\kappa} D^{\kappa}\right) Y(t)=g\left(1+\mu_{1} D+\cdots+\mu_{\lambda} D^{\lambda}\right) X(t-\tau)
$$

where:

$D=\mathrm{d} / \mathrm{d} t$ represents the first derivative with respect to time;

$Y(t)$ is a continuous function of time representing the output of a dynamical system;

$X(t)$ is a continuous function of time representing the input of a dynamical system;

$\tau$ is the so-called dead-time parameter allowing for a possible delay in the response of the output to a stimulus in the input;

$g$ is the so-called gain of the system, i.e. the long run proportionality constant between the input and the output;

$\pi_{1}, \cdots, \pi_{\kappa}$ and $\mu_{1}, \cdots, \mu_{\kappa}$ are constant coefficients.

Equation (2) can describe a wide range of mechanical, thermal, or economic systems (see for instance Box et al. [24]). Equation (1) is a generalization of equation (2) in discrete time in the sense that it incorporates several inputs (indicator series) of both random and non-random character. The gain for each input, though not explicitly included in Equation (1) is expressed as:

$$
g_{i}=\frac{\omega_{i 0}-\omega_{i 2}-\cdots-\omega_{i s_{i}}}{1-\delta_{i 1}-\cdots-\delta_{i r_{i}}}
$$

The $\omega_{i}(B)$ and equivalently and $\left.\omega_{j}^{\prime}(B)\right)$ polynomials reflect the total change in asset returns caused by the random indicator series $I_{i t}$ and the first difference of the non-random indicator $I_{j t}^{*}$ respectively, while the $\delta_{i}(B)$ and equivalently $\delta_{j}^{\prime}(B)$ polynomials express the rate at which asset returns approach a new equilibrium level due to the effect of $I_{i t}$ and $I_{j t}^{*}$ respectively. The term: $\frac{\Theta(B)}{\Phi(B)} \varepsilon_{t}$ in Equation (1) represents the way in which new information released by sources not included in (1), e.g. private information, is reflected in $R_{t}$. If such information is not fully reflected in $R_{t}$ at least one of the coefficients of $\Theta(B)$ and/or $\Phi(B)$ should be different than zero, except for a special case to be described in the next section.

It must be further clarified that the measurement level of the non-random indicators $I_{j t}^{*}$ is lower than that of $I_{i t}$ Indeed, $I_{j t}^{*}$ are nominal variables and the reason that they have been included in Equation (1) is that with such variables it is possible to express quantitatively the effect of various events (e.g. an announcement) in observed returns. The difference operator $(\nabla)$ in front of the non-random indicators has been included so as the effect of an event on returns cuts off after exactly $s^{\prime}$ time periods, where $s^{\prime}$ is the order of the corresponding $\omega^{\prime}(B)$ polynomial (more technically, the difference operator converts a step 
function represented by the non-random binary indicator into a pulse function), when the corresponding $\delta^{\prime}(B)$ polynomial equals 1$)$.

Finally, it is noted that for simplicity any error-correction terms have been assumed to be zero, hence, Equation (1) does not include such terms.

\section{Market Efficiency Testing}

\subsection{Simulating Market Conditions and Efficiency Testing}

An important advantage gained by the introduction of the return generating model, as formulated by Equation (1) and described in the previous section, is that by selecting certain combinations of parameter values in Equation (1), certain (simplified) market conditions may be simulated. Such market conditions can then be studied in conjunction with the established econometric approaches that are commonly used for the testing of the market efficiency hypothesis. In that way it will be possible to examine how the established approaches for market efficiency work, what is the outcome that they provide, and, whether or not, the later reflect the assumed market conditions.

\subsection{Weak Form Market Efficiency Testing}

In WFME, tests of any kind (for a review see for instance Fama [1] [9] [25] [26] focus exclusively on the information (if any) embodied in the history of returns, hence, such tests ignore any other information source. Inevitably, $\mathrm{R}_{\mathrm{t}}$ is necessarily parameterized in a univariate way i.e.

$$
R_{t}=\frac{\Theta^{\prime}(B)}{\Phi^{\prime}(B)} \varepsilon_{t}^{\prime}
$$

Therefore, with WFME tests, all dynamic impacts (due to public or even private information, see also para 3.4 below) which are responsible for the formation of observed returns are detected through the new autoregressive and/or moving average parameters of the univariate model.

Below some special cases of dynamic impacts on $R_{t}$ are examined; for simplicity it is assumed that $I_{j t}^{*}, j=1, \cdots, M$ will be equal to zero.

a) $s_{i}=r_{i}=b_{i}=0$

In this case the (simulated) market is obviously efficient. Further, for each time period the impact of information on $R_{t}$ will be equal to $\omega_{10} I_{1 t}+\omega_{20} \mathrm{I}_{2 t}+\ldots+$ $\omega_{\mathrm{k} 0} I_{k t}$. Hence, $R_{t}$ will be i.i.d. and any WFME test will, correctly, not reject the null hypothesis.

b) $s_{i} \neq 0$ for at least one $i=i^{\prime}, r_{i}=b_{i}=0, \forall i$

In this case information transmitted from the indicator series will not be fully reflected in $R_{t}$ simultaneously. At least $s_{i^{\prime}}$ time periods will be required for the full reflection of new information from $I_{i^{\prime} t}$ on $R_{t}$, so the market is inefficient. Moreover, simple trading rules or autocorrelation tests can capture this gradual assimilation of new information on $R_{t}$ hence, WFME tests will, correctly, reject the null hypothesis.

c) $s_{i} \neq 0, r_{i} \neq 0$ for at least one $i=i^{\prime}, b_{i}=0, \forall i$ 
The situation is the same as in case (b) with the only difference that in this case information from $I_{i^{\prime} t}$ will be fully reflected in $R_{t}$ theoretically after infinite time. Therefore, the market is inefficient with respect to the reflection of new information from $I_{i^{\prime} t}$ on $R_{t}$ and WFME tests will, correctly, reject the null hypothesis.

d) $s_{i}=r_{i}=0, \forall i, b_{i}=0 \forall i \neq i^{\prime}$ and $b_{i} \neq 0$ for $i=i^{\prime}$

Here the value $\omega_{i^{\prime} 0} I_{i^{\prime} t}$ will only be fully reflected in $R_{t}$ after $b_{i^{\prime}}$ time periods. However, though the market is clearly inefficient, there is no way that this inefficiency be detected by any univariate method. Indeed, in this case $R_{t}$ is i.i.d. (i.e. asset prices will follow a random walk $\left.{ }^{1}\right)$. Hence, any test for WFME will, falsely, conclude that the series of asset returns is totally unpredictable, whilst the series could be totally predictable, if the indicator series $I_{i^{\prime} t}$ were known.

(e) $s_{i} \neq 0, r_{i} \neq 0, b_{i} \neq 0$, for at least $i=i^{\prime}$

In this case, the market is apparently inefficient, and it is easily understood that WFME tests will indeed detect inefficiency (although they will understate its magnitude, as $b_{i} \neq 0$ ).

\subsection{Semi-Strong Form Market Efficiency Testing}

In the case of semi-strong-form market efficiency, at least one of the independent variables in Equation (1) must be assumed to be known. Letting this variable be a non-random one, then SSFME refers to event studies and the model described by Equation (1) becomes:

$$
R_{t}=\frac{\omega^{\prime}(B)}{\delta^{\prime}(B)} \nabla\left(I_{t-b}^{*}\right)+\frac{\Theta^{\prime \prime}(B)}{\Phi^{\prime \prime}(B)} \varepsilon_{t}^{\prime \prime}
$$

$I_{t-b}^{*}$ may be taken as a binary variable indicating the presence or absence of a particular event. The effect of all the omitted indicators is reflected in $\Theta^{\prime \prime}(B)$ and $\Phi^{\prime \prime}(B)$. It is noted that in the usual practice event studies are examined using cross sectional data of cumulative "abnormal" returns, where abnormal returns are calculated as the difference between observed and expected returns [1] [26]. The latter are determined using some equilibrium model for asset pricing. Aiming to the widening of the statistical procedures for SSFME testing, the approach will be somewhat different here.

In the case where $I_{t}^{*}$ is known, all the parameters of $\Theta^{\prime \prime}(B), \Phi^{\prime \prime}(B)$, $\omega^{\prime}(B), \quad \delta^{\prime}(B)$ can be simultaneously estimated by maximum likelihood [24]. So, Equation (3) (and Equation (1)) suggests that WFME and SSFME (with respect to $I_{t}^{*}$ and for a single asset) should be tested simultaneously.

The above approach may have applications in the empirical testing of some special forms of SSFME. At first it is noted that the effect of events of the same type that repeatedly occur for a single asset (e.g. earnings surprises) could be studied by a model such as the one expressed by Equation (4). As the occurrences of such an event may not be at consecutive time periods the values of the

${ }^{1}$ The usual terminology in finance, which will be followed, is somewhat loose with respect to this matter. It is emphatically noted that strictly speaking if asset returns are i.i.d. it is true that the log of prices are random walk, not prices themselves. 
$\omega^{\prime}(B)$ and $\delta^{\prime}(B)$ polynomials will reflect the average effect of such an event (see Milionis and Papanagiotou [27] for the technical details about the impact assessment of external factors acting discontinuously on autocorrelated time series). It is noted that with the established methodology, as briefly described previously, in order to minimize the effect of other events that may occur at the same time, the average effect across all firms is used. However, as Elton et al. [26] recognize, for studies where the magnitude of an announcement may vary considerably across firms it is necessary to examine individual firm behaviour as well. Yet, Elton et al. [26] do not suggest any specific way for doing so. The approach proposed just above is in line with the concern of Elton et al. [26] and could provide a way to cover this gap. Moreover, it is noted that when $\mathrm{s}$ and/or $\mathrm{r}>1$ (where $\mathrm{s}, \mathrm{r}$ represent the degree of the $\omega^{\prime}(B)$ and $\delta^{\prime}(B)$ polynomials respectively), it is apparent that with the proposed approach the effect of an event is in general not constant, but a function of the time elapsed after the occurrence of the event. Hence, the proposed approach offers a greater flexibility over a yes-or-no outcome and there is little doubt that this is an additional advantage of it. Further, Equation (4) could also be applied to particular types of events that for data of frequency of days ${ }^{-1}$ or higher could be characterised as very rare (e.g. a national currency devaluation, a severe terrorist attack) where the established methodology, as described above, cannot be used. More importantly, as the suggested model can accommodate several sources of information, semi-strong-form market efficiency may be tested with respect to the assimilation of new information, released from more than one source, simultaneously. An additional advantage of such an approach is that it is econometrically compatible with the need to eliminate specification bias (omitted variables) in any parameter estimation (for details about the so-called specification bias see any econometrics textbook). In that sense it is apparent that asset pricing models such as the static Capital Asset Pricing Model or conditional versions of it [16] can be hosted in the testing procedure.

\subsection{Strong-Form Market Efficiency Testing}

As is well known, strong-form efficiency in practice can be tested only indirectly [26]. In contrast to the other two cases where new information is assumed to be released simultaneously to all investors, private information is initially known only to a subgroup of investors (insiders) before this information becomes gradually available to all investors. Hence, if insiders decide to react on the basis of private information, such information can only be fully reflected in asset prices gradually.

However, within the stated framework, as the latter was quantitatively expressed by Equation (1), it is in theory possible to test for strong-form efficiency directly, assuming that: (a) it is possible to identify the effect on prices of all publicly available information; (b) the market is weak-form, as well as semi-strong-form efficient. Then SSME may be quantitatively defined by the condition that both 
the $\Phi(B)$ and $\Theta(B)$ polynomials in Equation (1) should equal 1. Consequently, any non-zero valued coefficient in either $\Phi(B)$ and/or $\Theta(B)$ polynomials in Equation (1) entails the rejection of strong-form efficiency. In practice, however, assumption (a) is very difficult to be fulfilled, as it is next to impossible to identify every single source of information that could potentially affect asset prices. How much more then, to identify the character of the effect of such a source on asset prices. Therefore, it is indistinguishable to find out, whether or not, any nonzero valued coefficient in either $\Phi(B)$ and/or $\Theta(B)$ polynomials in Equation (1) entails the rejection of SFME specifically, or the rejection of a lower type of efficiency.

At the other end of the spectrum, ignoring all sources of information, any testing procedure is restricted to past returns, as discussed already commenting on Equation (3). Using the same line of reasoning as above, even considering Equation (3) which was initially related to WFME, it is apparent that in reality any non-zero valued coefficient in either $\Phi^{\prime}(B)$ and/or $\Theta^{\prime}(B)$ polynomials in Equation (3) may not entail the rejection of exclusively weak-form efficiency ${ }^{2}$ as it could also be attributed to the gradual assimilation of private information, as discussed above. That seems to bring market efficiency, at least in its evolving version which is not incompatible with the existence of trends and bubbles in market dynamics (Lo, 2004), closer to its alleged opponent, i.e. the so-called Technical Analysis ${ }^{3}$. Indeed, in the heart of the Technical Analysis approach is the belief that market action discounts everything. That means that according to Technical Analysis, markets react not only to the known fundamentals, but also to the unknown fundaments [28], which are obviously related to private information. It is clearly stated however, that further elaboration is required to substantiate this argument more concretely.

\section{Further Comments}

1) The efficiency testing based on a dynamic model expressed as a special case of Equation (1) is compatible with the general notion of causality [29]. Indeed, using this model, the effect of any external factor (event) on asset returns is significant if part of the series variance initially attributed to the stochastic component of the model, after the inclusion of a deterministic component (an indicator series) is attributed to the indicator series, i.e. the external factor.

2) In the literature thus far, a great deal of effort has been spent in order to show that univariate models for asset prices less restrictive than the random walk may be compatible with market efficiency [8]. Pure random walks are unquestionably related with efficient markets, and, usually, no further investigation is undertaken in relation to market efficiency, once asset prices are found to follow a random walk process [30] [31]. The market is considered as efficient in

${ }^{2}$ In any case, the existence of a non-zero coefficient in $\Phi^{\prime}(\mathrm{B})$ and/or $\Theta$ (B) means the certain rejection of efficiency, as constancy of expected returns has been assumed.

${ }^{3}$ By the term "Technical Analysis" is meant the study of market action, using charts and other tools and practical rules, aiming at forecasting future price trends. 
general, not just at the weak form level. As Peters [32] quotes "...Market efficiency does not necessarily imply a random walk, but a random walk does imply market efficiency..." However, as shown in the previous section (case (d)), a pure random walk in asset prices does not necessarily entail market efficiency. Further, to the best of the author's knowledge there is no work in which efficiency was tested and rejected by "event studies" and at the same time the series of asset prices was found to be a pure random walk. Hence, existing tests for SSFME are rather tests on whether departures from the pure random walk in prices are due to real inefficiency or due to the joint hypothesis problem.

3) It is noteworthy that, in general, cross sectional correlations of returns have been found to exist in empirical research. A clear evidence of cross correlations is the positive autocorrelation found in the returns of many price indices formed by stocks which, individually, appear to have negative autocorrelation in their returns (see for instance Campbell et al. [25] who also note that the nature and sources of such cross-sectional predictability are unclear). However, caution is needed on how these empirical results are related to case (d) of the previous section. Lead-lag patterns found empirically in asset returns may be attributed to friction in the trading process (e.g. non-synchronous trading, see Cohen et al.. [33]). This situation refers to observed returns, while case (d) of the previous section, which is purely theoretical, refers to true returns. In observed returns price adjustment delays create not only cross-correlations among asset returns, but also, at the same time, autocorrelations. In contrast, case (d) of the previous section refers to a lead-lag relationship between two iid series.

\section{Summary and Conclusions}

A discrete time return generating model is proposed in this work. The model assumes a mechanism by which new information is released and assimilated in asset prices. This mechanism is a simplification of reality, but nevertheless it can make a useful tool for an assessment of market efficiency testing approaches.

Although financial econometricians have tried to reconcile stochastic processes for asset prices, which are less strict than the random walk, with an efficient market, a pure random walk in prices has been taken for granted to imply efficient markets. This is possibly the reason that often in the existing empirical work for SSFME testing no further tests follow, after a random walk has been detected in asset returns. SSFME testing is performed independently from WFME testing. In this work, simulating various market conditions by using various combinations of model parameters it was shown that, theoretically, there exists a case where markets in which prices follow a pure random walk, are at the same time perfectly predictable. A distinction is made between these theoretical findings, which refer to true returns, and cross predictability of observed returns, which is attributed to friction in the trading process and is accompanied by autocorrelation in the series of returns as well.

Furthermore, the theoretical return generating model for asset returns sug- 
gested in this work may have practical applications in the empirical testing of semi-strong form market efficiency for some special cases and in that way could cover an existing gap in the SSFME testing, as discussed in Section 3. Such cases comprise rare events, events which occur repeatedly for the same asset, or even combinations of events with the possible inclusion of other pieces of new information quantitatively expressed at a higher measurement level. Finally, commenting on strong-form efficiency testing, it was insinuated that the notion of market efficiency, particularly in its evolving version, may not be constantly incompatible with Technical Analysis, as it is believed.

\section{Conflicts of Interest}

The author declares no conflicts of interest regarding the publication of this paper.

\section{References}

[1] Fama, E.F. (1970) Efficient Capital Markets: A Review of Theory and Empirical Work. The Journal of Finance, 25, 383-417. https://doi.org/10.2307/2325486

[2] Roberts, H. (1967) Statistical versus Clinical Prediction of the Stock Market CRSP. University of Chicago, Chicago.

[3] Rubinstein, M. (1975) Securities Market Efficiency in an Arrow-Debreu Economy. American Economic Review, 12, 812-824.

[4] LeRoy, S.F. (1976) Efficient Capital Markets: Comment. The Journal of Finance, 31, 139-141.

[5] LeRoy, S.F. (1989) Efficient Capital Markets and Martingales. Journal of Economic Literature, 27, 1583-1621.

[6] Malkiel, B. (1992) Efficient Market Hypothesis. In: Newman, P., Milgate, M. and Eatwell, J., Eds., New Palgrave Dictionary of Money and Finance, McMillan, London.

[7] Milionis, A.E. (2007) Efficient Capital Markets: A Statistical Definition and Comments. Statistics and Probability Letters, 77, 607-613. https://doi.org/10.1016/j.spl.2006.09.007

[8] Milionis, A.E. and Moschos, D. (2000) On the Validity of the Weak-Form Efficient Markets Hypothesis Applied to the London Stock Exchange: Comment. Applied Economics Letters, 7, 419-421. https://doi.org/10.1080/135048500351087

[9] Fama, E.F. (1991) Efficient Capital Markets: II. The Journal of Finance, 46, 15751617. https://doi.org/10.1111/j.1540-6261.1991.tb04636.x

[10] Al-Loughani, N. and Chappell, D. (1997) On the Validity of the Weak-Form Efficient Markets Hypothesis Applied to the London Stock Exchange. Applied Financial Economics, 7, 173-176. https://doi.org/10.1080/096031097333736

[11] Shmilovici, R., Alon-Brimer, Y. and Hauser, S. (2003) Using a Stochastic Complexity Measure to Check the Efficient Market Hypothesis. Computational Economics, 22, 273-284. https://doi.org/10.1023/A:1026198216929

[12] Squalli, J. (2006) A Non-Parametric Assessment of Weak-Form Efficiency in the UAE Financial Markets. Applied Financial Economics, 16, 1365-1373. https://doi.org/10.1080/09603100500447594

[13] DePenya, F.J. and Gil-Alana, L.A. (2007) Serial Correlation in the Spanish Stock 
Market. Global Finance Journal, 18, 84-103.

https://doi.org/10.1016/j.gfj.2007.01.001

[14] Mollah, A.S. (2007) Testing Weak-Form Market Efficiency in Emerging Market: Evidence from Botswana Stock Exchange. International Journal of Theoretical and Applied Finance, 10, 1077-1094. https://doi.org/10.1142/S021902490700455X

[15] Milionis, A.E. and Papanagiotou, E. (2009) A Study of the Predictive Performance of the Moving Average Trading Rule as Applied to NYSE, the Athens Stock Exchange and the Vienna Stock Exchange: Sensitivity Analysis and Implications for Weak-Form Market Efficiency Testing. Applied Financial Economics, 19, 1117 1186. https://doi.org/10.1080/09603100802375519

[16] Milionis, A.E. and Papanagiotou, E. (2011) A Test of Significance of the Predictive Power of the Moving Average Trading Rule of Technical Analysis Based on Sensitivity Analysis: Application to the NYSE, the Athens Stock Exchange and the Vienna Stock Exchange. Implications for Weak-Form Market Efficiency Testing. Applied Financial Economics, 21, 421-436. https://doi.org/10.1080/09603107.2010.532105

[17] Lim, K.P. and Brooks, R. (2011) The Evolution of Stock Market Efficiency over Time: A Survey of the Empirical Literature. Journal of Economic Surveys, 25, 69-108. https://doi.org/10.1111/j.1467-6419.2009.00611.x

[18] Daniel, K. and Titman, S. (1999) Market Efficiency is an Irrational World. Financial Analysts Journal, 55, 28-40. https://doi.org/10.2469/faj.v55.n6.2312

[19] Lo, A.W. (2004) The Adaptive Markets Hypothesis: Market Efficiency from an Evolutionary Perspective. The Journal of Portfolio Management, 30, 15-29. https://doi.org/10.3905/jpm.2004.442611

[20] Lo, A.W. (2005) Reconciling Efficient Markets with Behavioural Finance: The Adaptive Markets Hypothesis. Journal of Investment Consulting, 7, 21-44.

[21] Grossman, S.J and Stiglitz, J.E. (1980) On the Impossibility of Informationally Efficient Markets. American Economic Review, 70, 393-408.

[22] Hou, K. and Moskowitz, T.J. (2005) Market Frictions, Price Delay, and the Cross-Section of Expected Returns. The Review of Financial Studies, 18, 981-1020. https://doi.org/10.1093/rfs/hhi023

[23] Peters, L. (1989) Fractal Structure in the Capital Markets. Financial Analysts Journal, 45, 32-37. https://doi.org/10.2469/faj.v45.n4.32

[24] Box, G.E.P., Jenkins, G.M. and Reinsel, G.C. (1994) Time Series Analysis Forecasting and Control. 3rd Edition, Prentice-Hall, Upper Saddle River, NJ.

[25] Campbell, J.Y., Lo, A.W. and Mackinlay, A.C. (1997) The Econometrics of Financial Markets, Princeton University Press, Princeton, NJ.

[26] Elton, E.J., Gruber, M.J., Brown, S.J. and Goetzman, W.N. (2007) Modern Portfolio Theory and Investment Analysis. 7th Edition, Wiley, New York.

[27] Milionis, A.E. and Papanagiotou, E. (2009) An Alternative Methodological Approach to Assess the Predictive Performance of the Moving Average Trading Rule in Financial Markets: Application to the London Stock Exchange. Bank of Greece, Working Paper No.107.

[28] Murphy, J.J. (1999) Technical Analysis of the Financial Markets, a Comprehensive Guide to Trading Methods and Applications. New York Institute of Finance, USA.

[29] Yantek T. (1984) Box-Jenkins Transfer Function Analysis. Political Methodology, 10, 377-411.

[30] Huber, P. (1997) Stock Market Returns in Thin Markets: Evidence from the Vienna 
Stock Exchange. Applied Financial Economics, 7, 493-498.

https://doi.org/10.1080/096031097333358

[31] Laopodis, N.T. (2004) Financial Market Liberalization and Stock Market Efficiency: Evidence from the Athens Stock Exchange. Global Finance Journal, 15, 103-123. https://doi.org/10.1016/j.gfj.2004.06.001

[32] Peters, L. (1991) Chaos and Order in the Capital Markets. John Wiley \& Sons, Hoboken, NJ.

[33] Cohen, C., Hawawini, G., Maier, F., Schwartz, R. and Whitcomb, D. (1983) Friction in the Trading Process and the Estimation of Systematic Risk. Journal of Financial Economics, 12, 263-278. https://doi.org/10.1016/0304-405X(83)90038-7 